\title{
An improved SIFT algorithm based on canny operator and hillbert - huang transform
}

\author{
Deng-Shan Huang, Xiao-Nan Wang and Qi Qin \\ Department of Electronics and Engineering, Northwestern Polytechnical University, \\ Xi'an, China \\ E-mail:1036686063@qq.com
}

\begin{abstract}
An improved SIFT algorithm, based on Canny operator and Hillbert - Huang transform is put forward. Canny operator can effectively avoid the edge response but there will be a smooth transition and shift of the edge. We utilize Hillbert-Huang transform to enhance image contour. Then we propose an improved SIFT algorithm based on Canny operator and Hillbert-Huang transform. We compare the feature points extracted with original SIFT algorithm; we compare the feature points with the improved SIFT algorithm, which are smaller in number than those extracted with the original SIFT algorithm, eliminating unsatisfactory feature points and this giving better accuracy rates in Fig.1:19.7\% in the first case, $18.06 \%$ in the second case, and $19.43 \%$ in the third case.

Keywords: Canny Operator; Hillbert - Huang Transform; Improved SIFT(Scale Invariant Feature Transform) Algorithm.
\end{abstract}

\section{Canny and Hillbert-Huang}

In the field of industrial, agricultural, commercial and military, image matching, is an indispensable and important means for the research. In recent years, it has been to explore unceasingly. At home and abroad, there are mainly the following means: color or grayscale texture edge feature extraction, image algebra feature extraction, image transform coefficient feature extraction [1]. Among them, the SIFT algorithm is affected by the light intensity small, and it makes the target block processing result good. Also it has good robustness, with the characteristics of the high speed. In the feature point detection, SIFT is widely used by many scholars.

In literature [2], SIFT algorithm is put forward by some step: determining scale space, testing location of the selected point, determining the position of key and describing several key steps. Finally it realizes the image matching. However, sift algorithm is also flawed. Using DOG operator for edge detection, the SIFT algorithm get the key with the edge response.So there are a lot of points are not really key points. 
In the edge detection, the Canny operator has some advantages, such as the optimization of filter, enhancement and testing. And It has better capability against of noise. However, in the processing step of Canny operator, using Gaussian operator to smooth image will make excessive smoothing and edge move [3]. In the image decomposition, there is a method named Twodimensional Hillbert - Huang transform. The image can be decomposed into corresponding intrinsic mode function, and Two-dimensional Hillbert - Huang transform make the edge profile more clear. So it combine Canny with Hillbert Huang transform, and then detect the edge points. At last, it will get the edge points, and make the points compare with SIFT algorithm of edge points. The points which have been got by both Canny detection and Hillbert-Huang detection compare with the points detected by SIFT. The key points is detected by both the first detection and the second detection, namely to choose feature points.

John f. Canny put forward Canny edge detection operator in 1986, it is recognized as the most excellent edge detection algorithm in the image processing field. And it has good anti-noise performance, so experts improve and research it. Canny operator edge detection is divided into five steps:

(1)Using Gaussian filter smooth and filter the image

$$
H(x, y)=G(x, y) * I(x, y)=G(y) *(G(x))
$$

Wherein, $G(x)$ is the horizontal direction, $G(y)$ is the vertical direction $G(x, y)$ is the Gaussian filter, $I(x, y)$ is the original image, $H(x, y)$ is the image smoothed.

(2) calculate the strength and direction of the image.

$$
\begin{gathered}
P_{x}(i, j)=(I[i+1, j] I[i, j]+I[i+1, j+1] \quad I[i, j+1]) / 2 \\
P_{y}[i, j]=(I[i, j+1] I[i, j]+I[i+1, j+1] \quad I[i+1, j]) / 2 \\
M[i, j]=\sqrt{P_{x}[i, j]^{2}+P_{y}[i, j]^{2}} \\
{[i, j]=\arctan \left(P_{y}[i, j] / P_{x}[i, j]\right)}
\end{gathered}
$$

Among them, (2) (3) is the partial derivatives, (4) (5) is of the magnitude and direction of the gradient. 
(3) Using non maxima suppression to eliminate the error points and obtain single pixel edge points.

In the $3 \times 3$ neighborhood of image, if the gradient values of two adjacent points below the current point, this point can be considered as an edge point, marked 1 , otherwise labeled 0 .

(4) Using bi-threshold binarization to obtain boundary.

Define level threshold which is needed to detect. Gradient magnitude greater than the high threshold is invariably edge, and gradient value which is below the low threshold is certainly not at the edge. In between, it is determined whether there are eight neighborhood above the high threshold gradient magnitude. If 8 neighborhood has gradient magnitude which is higher than high threshold, it is the edge.

(5) Make boundary track, resulting in a final edge image.

Linear smoothing with a Gaussian filter Is the more important step in Canny edge detection operator. If the value of Gaussian spatial is small, It will get high accuracy but poor smoothness and high noise. If you want to suppress the noise, you have to increase the value of the Gaussian space .It will cause serious edge position shift.

Two-dimensional Hillbert - Huang transform is an effective means of

Contemporary nonlinear non-stationary random signal. There is two steps: (1) Multi-resolution empirical mode decomposition (EMD) (2) instantaneous frequency solver. Dimensional Hilbert-Huang transform is applied to decompose the picture. According to the frequency of the level, it could decompose the signal of IMF. Combining the two-dimensional transform with Canny operator could extract edge of image and profile accurately. Specific steps are as follows: (1) Solving the IMF, and using two-dimensional HHT to decompose image. It will obtain the high frequency portion $\mathrm{H} 1$ which is contained the information and the low frequency which is contained contour.

$$
\begin{aligned}
& c_{1}=I(x, y) \quad m I(x, y) \\
& c_{2}=I(x, y) \quad c_{1} \quad m\left[I(x, y) \quad c_{1}\right]=m I(x, y) \quad m^{2} I(x, y) \\
& c_{3}=I(x, y) \quad c_{1} \quad c_{2} \quad m\left[I(x, y) \quad c_{1} \quad c_{2}\right]=m^{2} I(x, y) \quad m^{3} I(x, y) \\
& c_{n}=m^{n} I(x, y) \quad m^{n} I(x, y)
\end{aligned}
$$


Add (6)(7)(8)(9), it will get Two-dimensional IMF:

$$
I(x, y)={ }_{i=1}^{n} c_{n}+m^{n} I(x, y)
$$

$I(x, y)$ is a two-dimensional signal, for the signal to solve the mean surface, the $\mathrm{n}$-th component of the IMF.

(2)Continue to use the two-dimensional FFT to decompose the lowfrequency part, and get high-frequency part $\mathrm{H} 2$ and low-frequency part L1.

(3) Strengthen the contour of image.

$$
I=1.2 \quad L 2+0.4 \quad H 2
$$

(4)Using Canny operator to process the two-dimensional HHT transform and detecting the edge.

\section{Improved SIFT Algorithm}

SIFT (Scale Invariant Feature Transform) algorithm is first proposed by David G.Lowe. In 2004, it is improved. The core of SIFT algorithm is the theory of scale space invariant. And for illumination, occlusion, debris scene and image rotation, zooming, panning and other disturbances, there are better results. However, this algorithm is also defective. It is that when using a DOG operators to position the key points, it will produce a strong edge response. Although there is the step which is the SIFT eliminate the wrong point, we will get some wrong points. Improve the step which is the SIFT using DOG operator building pyramids to detect and obtained key points to use Canny edge detection and Hilbert-Huang transform access to key points.

(1)define the scale space and DOG operator.

$$
\begin{gathered}
L(x, y,)=G(x, y, \quad I(x, y) \\
G(x, y, \quad)=\frac{1}{22} e^{\frac{(x \mathrm{~m} / 2)^{2}+(y \mathrm{n} / 2)^{2}}{2^{2}}}
\end{gathered}
$$

$G(x, y$,$) is Gaussian function, I(x, y)$ is the original image, denotes the convolution operation. To detect key points of scale space effectively, David G. Lowe propose DOG (Difference of Gaussian) operator. It is obtained from the difference of Gaussian different scales and image convolution.

$$
D(x, y, \quad)=(G(x, y, k) \quad G(x, y, k)) * I(x, y)=L(x, y, k) \quad L(x, y, \quad)
$$


(2)Extreme point detection space

The key point is composed of the DOG of the local minima. The key point is to give a preliminary comparison between the two layers through the DOG in a group of adjacent. Any arbitrary pixel needs and compare its all adjacent points. We need to compare 3 3adjacent eight points in the field, as well as upper and lower levels, total $8+92=26$ points. Make difference which is obtained discrete point on the sub-pixel. And make the difference of the DOG curve fitting, Use Taylor expansions of DOG function on scale space. Let equation is zero, find the derivative, obtaining the corresponding extreme point equation.

$$
\begin{gathered}
D(x)=D+\frac{D^{T}}{X} X+\frac{1}{2} X^{T} \frac{{ }^{2} D}{x^{2}} X \\
X=\frac{{ }^{2} D^{1}}{X^{2}} \frac{D}{X} \\
D(\hat{X})=D+\frac{1}{2} \frac{\partial D^{T}}{\partial X} \hat{X}
\end{gathered}
$$

$X=(x, y,)^{T} X$ is centered offset. Any one dimension in $\mathrm{X}$ value exceeds 0.5 , it is considered an offset.

(3) Add Canny and Hilbert-Huang transform, to determine the final feature points.

Eliminate edge response, and get set of features R1 which is detected by SIFT algorithm. Add Canny and Hilbert-Huang transform to detection of image edge. It will obtain the set of feature points R2. Make comparison of feature points. If it is the same, we will keep the points of R2. If it is different, we will compare R2 with R3 which is 3 3points neighborhood of R2. If it is the same, we will keep the points of R2. If it is different, we will compare continuously.

$$
\begin{gathered}
f_{1}=R_{1} \quad R_{2} \\
f_{2}=\operatorname{size}\left(\left(\begin{array}{ll}
R_{3} & \left.R_{1}\right), 1
\end{array}\right)\right.
\end{gathered}
$$

$f_{1}$ and $f_{2}$ is landmarks, when $f_{1}$ is 0 , we could remove set of corresponding points. When $f_{1}$ is 1 , the points will be remained. When $f_{2}$ is 8 , remain the corresponding points in $R_{1}$. When $f_{2}$ is 7 , it could be removed.

(4)The description and distribution of key point. 
The amplitude and direction of the key point gradient:

$$
\begin{gathered}
m(x, y)=\sqrt{(L(x+1, y) \quad L(x \quad 1, y))^{2}+(L(x, y+1) \quad L(x, y \quad 1))^{2}} \\
(x, y)=\tan ^{1}\left((L(x, y+1) \quad L(x, y \quad 1)) /\left(L(x+1, y) L\left(\begin{array}{ll}
x & 1, y)))
\end{array}\right)\right.\right.
\end{gathered}
$$

To determining the calculation described region of space, it needs to calculate weight and generate a gradient histogram. According to the size of feature point, it could sort and generate the feature points.

\section{Result}

Apply the improved SIFT to image processing. Compared to the original SIFT algorithm, edge detection can be found on the number of points to reduce about $17 \%$ using 40 pictures in the experiment. Here are five pictures to experiment, using the original SIFT algorithm and the testing results of the improved SIFT algorithm:
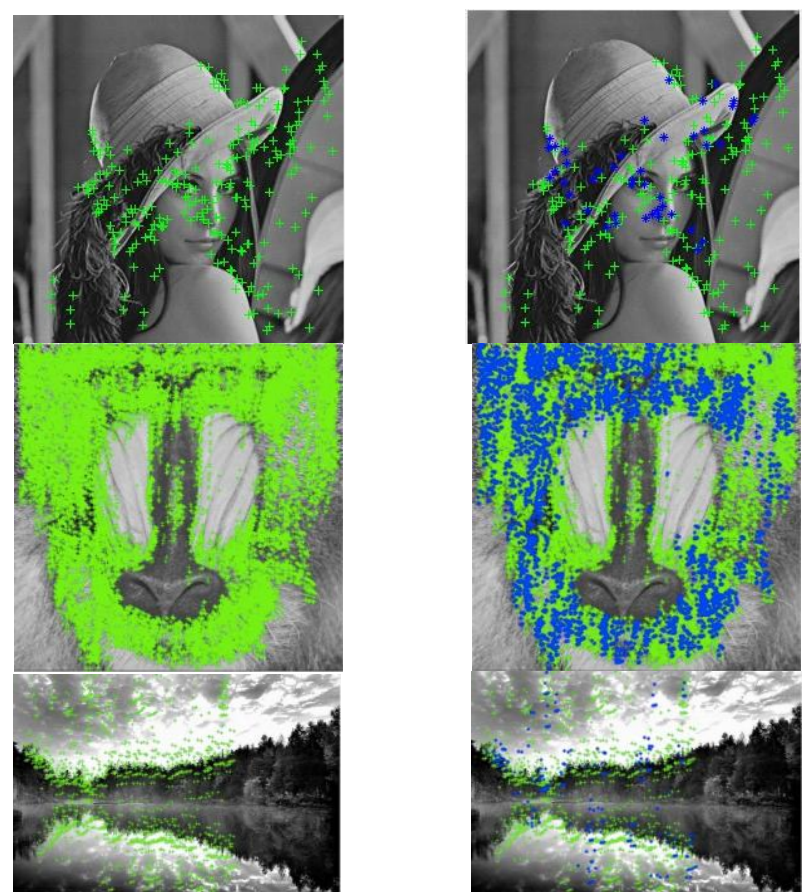

Fig. 1 This is the characteristic points

Green point in figure 1 is the original SIFT algorithm to detect the characteristic points, the blue point is the improved SIFT algorithm to remove 
edge points. Statistical the three pictures, table 1 can be obtained. Table 1 shows the feature point's number using the improved SIFT algorithm which is extracted. The picture 3 is complex, it will be many wrong points for edge response using the original SIFT. The improved SIFT algorithm not only increase the robustness, but also it can improve the accuracy.

Tab. 1 This is the feature point's number using the improved SIFT algorithm which is extracted

\begin{tabular}{cccc}
\hline Schedule & Picture 1 & Picture 2 & Picture 3 \\
\hline The points of original SIFT & 132 & 22929 & 1158 \\
The points of improved SIFT & 106 & 18788 & 933 \\
Remove points & 26 & 4141 & 225 \\
Accurate rate & $19.07 \%$ & $18.06 \%$ & $19.43 \%$ \\
\hline
\end{tabular}

\section{Conclusion}

The paper provides the algorithm is based on the original SIFT algorithm. Using the Canny and Hillbert - Huang transform for data fusion algorithm of edge detection method. By joining the comparison of feature points, it improves the accuracy of the feature point detection. The paper make the SIFT algorithm in image matching play a greater role. The proposed algorithm can remove edge points about $18 \% \sim 19 \%$, improving the stability and resistance to noise performance. This paper puts forward the improved algorithm can only be applied to two-dimensional gray image, the color image processing also needs further research.

\section{References}

1. Zhai Junhai, Zhao Wenxiu, Wang Xizhao. Research on the Image Feature Extraction[J]. Journal of Hebei University, 2009, 29(1): 106-112.

2. Lowe, D, G. Distinctive Image Features from Scale-Invariant Keypoints[J]. International Journal of Computer Vision, 2004, 60(2): 91-110C. Jarlskog, CP Violation (World Scientific, Singapore, 1988).

3. Li, Nan, Zhou, Bo. Edge deteciton of image based on Canny and 2demension Hilbert-huang transform[J]. ComPuter Englneering and Appilacations, 2011, 47(8): 209-211.

4. Zhao, Aigang, Wang, Hongli, Yang, Xiaogang, Lu, Jinghui1;, He, Xing1. Compressed sense SIFT descriptor mixed with geometrical feature[J]. Hongwai yu Jiguang Gongcheng, 2015, 3(44): 1085-1091. 
5. Chen, chunche, Shang-Lin. Using binarization and hashing for efficient SIFT matching $[\mathrm{J}]$. Journal of Visual Communication and Image Representation, 2015, (30): 86-93.

6. Wang, Anna, Wang, Zhe, Li, Shiyao. Research on a novel medical image non-rigid registration method based on improved SIFT algorithm[J]. Lecture Notes in Computer Science, 2010, (3): 91-99.

7. Qian, Sen, Zhu, Jianying. Improved SIFT-based Bidirectional Image Matching Algorithm[J]. Mechanical \& Electronic Engineering Institute, 2007, (9): 1179-1182. 